\title{
MJN REVIEW OF CULTURE ISSUES AND CHALLENGES AFFECTING NURSING PRACTICE IN SAUDI ARABIA
}

\author{
Abdulrhman Albougami, Jazi S Alotaibi* \\ Department of Nursing, College of Applied Medical Sciences, Majmaah University, Saudi Arabia \\ *Corresponding author's email: jalotaibi@mu.edu.sa
}

\begin{abstract}
Cultural diversity is a prominent issue in nursing practice in Saudi Arabia, which hinders the development of the profession. The presence of an expatriate nursing workforce in the region leads to issues of linguistic and cultural differences between nurses and patients. The literature reveals that conflicts between expatriate nurses and patients are caused primarily by a lack of effective communication and interaction, which, in turn, originates from differences in cultural standards. Such conflict may compromise patient care and increase the probability of medical errors. The concerned authorities must consider these issues when recruiting foreign-educated nurses. Educational and training programs must also include guidelines for cultural sensitivity so that nurses can competently deal with culturally delicate situations. Nurses must proficiently handle various aspects of cultural diversity among patients because such competencies are needed to provide effective care.
\end{abstract}

Keywords: Culture Issues, Challenges, Nursing Practice

\section{INTRODUCTION}

Several challenges in modern healthcare originate from social and technological issues and highly complex bioethical problems. Effectively addressing these challenges necessitates training that equips nurses with the proficiency essential to meet patient needs and enhance the quality of care (Almutairi, \& Mousa, 2014). The realization of healthcare goals, however, is complicated by workplace diversity, which is a constant in every professional field. Nurses and patients always exhibit differences in behaviour and perception (Campinha-Bacote, 2011; Darnell \& Hickson, 2015; Tucker, Roncoroni \& Sanchez, 2015). The same situation is true for Saudi Arabia, which is confronted with several nursing-related obstacles, particularly the accumulation of a foreigner-dominated workforce (Al-Homayan et al., 2013; AlYami \& Watson, 2014). Culture plays a major role in the nursing profession. In most cases, the lack of cultural sensitivity is a critical cause of misunderstanding between local patients and expatriate nurses, especially those from non-Arab countries (Felemban, O'Connor \& McKenna, 2014). Nurses need to understand the cultural setting of a given region because such comprehension helps them develop strong and effective relationships with their patients. In the process, nurses can offer optimum healthcare and reduce the potential risks associated with patient-nurse conflicts (De Beer \& Chipps, 2014; Tucker et al., 2015). This article discusses the history of nursing education in Saudi Arabia and evaluates the cultural facets of Saudi society and their effects on nursing practice.

Understanding the Structure and Role of the Saudi Society

The Saudi society is characterized by a reverence for the extended family, with each member exhibiting a sense of responsibility and affinity for parents and other relatives. The family is assumed to represent the individuality of a person. Saudis visit family members, celebrate their achievements, provide them with support, and show them compassion and respect (Alsulaimani, 2014). The family structure can be advantageous to nurses because they can interact easily and communicate with a patient's family members and involve them in a patient's care. Parents and grandparents occupy a position of considerable honour and respect, which gives them substantial authority over their offspring's healthcare; this authority also affects expectations regarding nursing services (AlKhathami et al., 2010). Patients are expected to be treated under nurses' care as they are treated at home - with respect, understanding, 
and compassion. Moreover, family members expect nurses to dress properly, assume a reserved disposition when interacting with patients, and avoid gestures or acts that may damage a patient's self-respect (Al Mutair et al., 2013). In Gulf countries, a person's honour is considered parallel to that of his or her entire family. Actions such as cruelty, sexually immorality and inappropriate behaviour, and mistreating the elderly or the weak tarnish the honour of an individual.

In family conversations, deaths and ailments are two of the most frequently discussed topics (Al-Shahri, 2002). When a family member is diagnosed with a disease, affiliation needed to increase with this member from the relatives. They must accompany patients when they visit the hospital for medical examinations or healthrelated interviews. In most cases, the family members accompanying the patient are the ones who communicate with the physician or other healthcare staff and respond on behalf of the patients. Such conduct is often frowned upon and prohibited by nurses on duty (AlKhathami et al., 2010).

Good care is measured by allowing elderly patients to stay close to their relatives in all the phases of their illnesses. Family members demand that patients be provided with the best possible healthcare, thereby demonstrating their concern for the elderly. Like the case of other family members, the elderly is accompanied by relatives during important stages of their treatment, such as follow-up consultations (Al Mutair et al., 2013).

Saudis attach considerable significance to family bonds and appreciate their families' efforts to satisfy their affiliation-related needs. When suffering from a disease or illness, Saudis tend to rely on these bonds to help facilitate healing. Individuals who are not visited as often by their families tend to feel lonely and rejected. In comparison to family and friends, nurses are considered outsiders by patients. Researchers have posited that the relationship between patients and nurses can improve when the nurse can interact with the patient in the same manner as families do. This level of involvement is regarded as more effective and compassionate than a professional approach (Felemban et al., 2014). Saudis tend to develop trust in nurses who care for their relatives compassionately and endeavour to know their families on a personal level. Nurses, for their part, are predisposed to sharing patient information with family members because such behaviour earns their trust. They must satisfactorily answer all the questions of family members so that nurses can reciprocate and refrain from withholding essential health-related information(Alshaikh et al., 2015).

\section{Understanding Gender Segregation}

Gender segregation is a common social norm practised by various government departments in Gulf countries. Males and females are prohibited from intermingling in open or public spaces, such as major hospitals and clinics. Specific zones are delineated for families, females, and males. In most social settings, career women are forbidden from working alongside or freely interacting with their male colleagues unless such interaction is crucial to a particular situation (Lamadah \& Sayed, 2014). For those who choose to carve out a career as a nurse, gender segregation remains a principal challenge encountered by teams who implement nursing assignments. They are required to assemble groups that comprise people of a single gender. Gender segregation can be maintained in professions such as education, but the same cannot be said of the nursing profession because nurses typically work alongside patients, physicians, and individuals of the opposite gender. Consequently, most nurses opt to offer services to patients of their gender (AlFozan, 2013). In the case of emergency nurses, however, caring for patients of the opposite gender is allowed. The societal norm of maintaining separation between men and women discourages most Saudi females from pursuing nursing as a profession.

Regardless of the prestige associated with nursing in the healthcare field and the promising career development that this discipline offers, many Saudi families are reluctant to allow their female members pursue this vocation because it cannot guarantee gender segregation in the workplace (Al-Homayan et al., 2013). Female nurses may fall into disgrace and risk the honour that society confers on their families (Al-Mahmoud, Mullen \& Spurgeon, 2012).

\section{History of Nursing Education in Saudi Arabia}

In 1958, the first Saudi nursing program was initiated when 15 Saudi males enrolled in a one-year nursing program. Over time, similar programs were offered to females, beginning in Riyadh and Jeddah. Admission to nursing programs initially depended on whether candidates completed fifth- or sixth-grade education. In 1981, this requirement was raised to mandatory ninthgrade education for the eligibility to enrol in an expanded three-year nursing education program (Aldossary, While \& Barriball, 2008; AlYami \& Watson, 2014).

In the 1970s, the Bachelor of Science in Nursing (BSN) program was launched, whereas master's-level programs were launched in 1987. In the beginning, all BSN programs were offered solely to females. The year 
2005 saw the launch of the first male BSN program, which reportedly had more than 300 male students registered in a five-year academic program in Riyadh (Ministry of Health, 2012). Given that the Saudi healthcare system are built in accordance with Western design, it has become necessary to westernize healthcare facilities and related educational institutions (AlMahmoud, Mullen \& Spurgeon, 2012). The problems encountered in the nursing profession in the Arab region reflected the cultural bias that is reinforced by the curricula and courses. The curricula indicate the significance of cultural diversity, but in practice, the Western culture is generally given priority (Almutairi \& McCarthy, 2015).

Generally, models of nursing education are considerably affected by these cultural variabilities. In the United States, for instance, baccalaureate nursing graduates are required to develop the knowledge and proficiency necessary to effectively serve a diverse population. This requirement compels them to understand the various dimensions of culture, religion, race, and gender, as well as the effect of these factors on the delivery of healthcare services (Darnell \& Hickson, 2015; Reyes, Hadley \& Davenport, 2013). The nursing staff must be educated on these issues because they may encounter stress from facing circumstances where culture shock occurs.

\section{The Nursing Practice}

The shortage of nurses has been a constant problem throughout the world, driving many skilled nurses to practice abroad, where they are offered more desirable working conditions and incomes. Moving from one place to another also affords nurses multicultural experiences (Lamadah \& Sayed, 2014; Rooyen, Telford \& Strümpher, 2010). In Saudi Arabia, the possibility of acquiring such experiences is significantly high, given that most of the nurses who serve in the country are expatriates with different cultural backgrounds. Nurses who practice in this region are confronted with a range of issues pertinent to local customs, healthcare practices, language, and communication (Almutairi \& McCarthy, 2015; Hussein, 2014).

Nursing is not considered one of the most desirable professions in Saudi society. Negative perceptions about the career, gender-based limitations, and an immense increase in the population have heightened the demand for expatriate nurses (Al-Fozan, 2013; Al-Mahmoud, Mullen, \& Spurgeon., 2012). In 2012, the percentage of expatriate nurses working in the country was estimated to be $63 \%$ of the total nursing population. They hail mainly from India, Malaysia, South Africa, Canada, the Philippines, New Zealand, and South Africa, as well as from Middle Eastern countries (Ministry of Health, 2012). The differences in professional, social, and cultural backgrounds are manifested in all levels of interaction - within expatriate nurses, between expatriate nurses and Saudi nurses, and between expatriate nurses and Saudi patients. Studies have confirmed that expatriate nurses faced major issue of having to satisfy the cultural needs of their patients (Al Momani \& Al Korashy, 2012; Almutairi \& McCarthy, 2015; Al Neami, Dimabayao \& Caculitan, 2014). A suggested approach to achieving this goal is to consult professional negotiators to work with practising nurses and help them overcome the issues associated with providing healthcare services to local patients. Negotiators or translators are not required to have nursing experience or training, but they must have considerable experience living within Saudi society. They serve as agents of culture, developing connections among different subcultures. They translate and interpret cultural symbols and language styles that characterize communication, values, and lifestyles; assistance from these agents that significantly facilitates the delivery of healthcare services (Almutairi \& McCarthy, 2015).

Time, context, and environment are the other facets of multicultural settings that are considered when examining the interaction between the Saudi public and expatriate nurses. Intense and strongly bonded relationships make the Saudi culture unique. In this cultural setting, events are evaluated with respect to the context that surrounds a relevant situation (Aldossary, 2013). As perceived by nurses, Saudis are invested in learning about a person and are always eager to form new relationships; however, this evaluation disregards an entire context and is based only on a current situation (Rooyen et al., 2010). Although Saudis are normally enthusiastic about conversing and interacting, they are strongly averse to engaging in such behaviour during crises, severe illnesses, disasters, and looming death. They tend to remain in denial during a crisis, which contrasts with Westerners' tendency to showpredilection for showing interest in every matter that is relevant to a situation (Al-Shahri, 2002). This denial is a principal obstacle to the effectiveness of healthcare staff, especially nurses. Being Muslims, Saudis have strong faith in divine aid, even in the most severe situations. They firmly believe that hope and faith help patients fight illness (Al Mutair et al., 2013).

Accordingly, Saudis regard the act of informing patients about their diseases as unkind. In an effort to 
refrain from hurting their loved ones, patients' relatives filter information about the patient's condition or completely withhold it from the afflicted family. This act is justified as a means of saving loved ones from potential emotional harm. Saudis assume that being aware of all the details of an illness causes a patient to lose hope (Alshaikh et al., 2015). In Saudi Arabia, nurses and other healthcare staff usually communicate such information indirectly (e.g., using non-verbal methods), tactfully avoiding reporting serious findings to patients and their families (Al Mutair et al., 2013).

\section{Cultural Diversity}

The rapid population growth in Saudi Arabia has increased the need for healthcare facilities and nursing staff. Owing to the ongoing social, technological, governmental, and economic changes in the country, the nursing profession has undergone various changes in the past years, thus affecting the provision of healthcare services in the region (Almalki, Fitzgerald \& Clark, 2011). Cohesion between different groups advances the development of an effective organizational structure that facilitates healthcare provision. In the Saudi nursing sector, this structure is highly complex because it depends heavily on a foreign workforce-a reliance that engenders conflicts in the healthcare field (Almutairi \& McCarthy, 2015; Zakari, Al Khamis \& Hamadi, 2010). The facilitation of effective healthcare services is often hindered by cultural diversity because it creates problems for nurses, who are required to communicate and interact with patients of different linguistic and cultural backgrounds. Healthcare staff should thoroughly understand cultural diversity to competently operate under different cultural contexts (AlKhathami et al., 2010).

\section{Cultural Sensitivity}

According to Campinha-Bacote (2011), cultural care is manifested by addressing the variabilities and commonalities of ethical standards, principles, and ways of life. Cultural sensitivity entails consideration for dissimilarities in culture, race, gender, and social class when dealing with a range of circumstances. Hussein (2014) indicated that the risk of culture shock arises in situations where nurses and patients have different cultural backgrounds. These settings may create an environment for more common nursing errors. Although Arabs and Westerners share several cultural values (e.g., attaching significance to bonds with family and children, aiming for a peaceful life), the variances in their traditions and histories overshadow these commonalities and tend to create conflicts, particularly in the healthcare field.

Felemban et al. (2014) described examples of how cultural sensitivity affects the caregiving process. Limited physical contact with patients may increase the risk of mistakes that practising nurses will make during healthcare delivery due to cultural and linguistic barriers. Such situations considerably affect the quality of care being offered.

The care planning process also differs across cultures. A patient may not comprehend matters such as the discontinuation of treatment, early discharge from the hospital, or refusal of hospital admission. In Saudi Arabia, ailments, apart from being medical conditions, are considered a "test" from Allah that a patient must endure with his or her faith intact for the duration of an illness (Felemban et al., 2014). By contrast, Western communities may deal with such issues through discussions and the consideration of alternatives. Healthcare delivery in Saudi Arabia may be affected by the lack of cultural sensitivity of healthcare staff, the social status of patients, the lack of affordable healthcare, and the unsuitability of services for different groups. As identified in the context of numerous countries, different cultural groups, to a certain extent, underutilize healthcare services (Ingram, 2012).

\section{Discussion of Potential Conflicts}

In the nursing profession, conflict may lead to waste of time and energy, distress, and confusion. Cultural insensitivity may generate conflict in different ways. AlFozan (2013) identified the factors that obstruct culturally competent care in Saudi Arabia as the inability to understand other cultures, inability to communicate effectively, diverse linguistic and cultural backgrounds of nurses, and the inability of healthcare organizations to address culturally diverse patients.

In addition, no industry standards are in place for the regulation of the nursing profession. Hence, organizations are compelled to develop their strategies for monitoring the nursing practice in general and the roles of nurses in particular; this individual development of strategies enables hospital authorities to address relevant issues (Aldossary, 2013; AlYami \& Watson, 2014; Zakari, Al Khamis \& Hamadi, 2010)

\section{Recommendations for Nursing Practice}

The current era is characterized by highly diverse workplaces, especially in the healthcare field. Patients may differ considerably from nurses in terms of cultural 
identity, religion, values, and beliefs, thus giving rise to diversity-induced complexity. Nurses are expected to be knowledgeable about the cultural backgrounds of their patients to ensure optimum care that corresponds with the cultural requirements. Such proficiency is called cultural competence - that is, the combination of approaches, strategies, and attitudes that enables nurses and other healthcare staff to work efficiently within transcultural settings (Bauce, Fitzpatrick, \& McCarthy, 2014; Harnegie, 2017).

In this context, cultural competence entails respect for cultural principles and the appraisal of cross-cultural relations. It ascribes importance to awareness about cultural variation dynamics, the growth of cultural information, and the modelling of services aimed at fulfilling special cultural needs (Campinha-Bacote, 2011). Nurses are duty-bound to grasp the cultural differences among their patients, but every patient must be treated with a similar level of care and compassion (Noble \& Rom, 2014). Several Saudi studies have shown that expatriate nurses are inadequately aware of the cultural knowledge that significantly affect nursing practice in the country (Al Momani \& Al Korashy, 2012; Almutairi \& McCarthy, 2015; Al Neami et al., 2014). Such factors must be brought to the attention of expatriate nurses applying for work in Saudi Arabia on their recruitment and orientation. This approach would significantly improve the standard of healthcare offered to patients. Nurses would therefore be substantially attentive to matters of cultural importance and distinctiveness (Rooyen, Telford \& Strümpher, 2010). Another imperative is for them to understand the diseases specific to Saudis and their culturally dictated care practices. Modern approaches to cultural sensitivity require that nurses evaluate their own cultural influences, beliefs about healthcare, biases, and heritage (Al Momani \& Al Korashy, 2012; Almutairi \& McCarthy, 2015).

Nurses may use existing literature or participate in training sessions and discussions to strengthen their knowledge about cultural sensitivity in patient care. They should extensively develop the skills required to recognize and grasp cultural differences among patients and engage in cross-cultural interactions. Another equally valuable recommendation is for nurses to exercise self-motivation in familiarizing themselves with the cultural attributes of Saudi Arabia and its citizens.

\section{CONCLUSION}

According to the distinct cultural setting of Saudi Arabia, the cultural issues faced by the country's nursing profession necessitate an effective resolution. This article discussed the history of nursing education in the country and evaluated the cultural facets of Saudi society as well as their effects on nursing practice. Nurses must endeavour to comprehend issues of cultural diversity among their patients to effectively deal with them. This skill will enhance their efforts to provide effective care for their patients.

\section{REFERENCES}

Al Khathami, A.M., Kojan, S.W., Aljumah, M.A., Alqahtani, H. \& Alrwaili, H. (2010). The effect of nurse-patient language barrier on patients' satisfaction. Saudi Medical Journal, 31, pp 1355-1358.

Al Momani, M. \& Al Korashy, H. (2012). Patient experience of nursing quality in a teaching hospital in Saudi Arabia. Iranian Journal of Public Health, 41(8), pp 42-49.

Al Neami, I., Dimabayao, G. \& Caculitan, E. (2014). Satisfaction level of inpatients on the nursing care provided by staff nurses at King Fahd Central Hospital. Journal of Nursing and Health Science, 3(2), pp 22-31.

Aldossary, A. (2013). The role legitimacy of nurses in Saudi Arabia. Journal of Health Specialties, 1(1), pp 28-37.

Aldossary, A., While, A. \& Barriball, L. (2008). Health care and nursing in Saudi Arabia. International Nursing Review, 55, pp 125-128.

Al-Fozan, H. (2013). Patients and family caregivers' satisfaction with care delivered by Saudi nurses at National Guard Health Affairs hospitals in Saudi Arabia. Journal of Natural Sciences Research, 3(12), pp 67-74.

Al-Homayan, A., Shamsudin, F., Subramaniam, C. \& Islam, R. (2013). Analysis of health care system: Resources and nursing sectors in Saudi Arabia. Advances in Environmental Biology, 7(9), pp 2584-2592.

Al-Mahmoud, S., Mullen, P. \& Spurgeon, P. (2012). Saudisation of the nursing workforce: Reality and myths about 
planning nurse training in Saudi Arabia. Journal of American Science, 8(4), pp 369-379.

Al-Mahmoud, S., Mullen, P. \& Spurgeon, P. (2012). Saudisation of the nursing workforce: Reality and myths about planning nurse training in Saudi Arabia. Journal of American Science, 8(4), pp 369-379.

Almalki, M., Fitzgerald, G. \& Clark, M. (2011). Health care system in Saudi Arabia: An overview. Eastern Mediterranean Health Journal, 17(10), pp 784-793.

Al-Mutair, A.S., Plummer, V., O'Brien, A. \& Clerehan, R. (2013). Family needs and involvement in the intensive care unit: a literature review. Journal of clinical nursing, 22(13-14), pp 1805-1817.

Almutairi, A. \& McCarthy, A. (2015). Understanding Cultural Competence in a Multicultural Nursing Workforce: Registered nurses' experience in Saudi Arabia. Journal of Transcultural Nursing, 26(1), 16-23.

Almutairi, K. \& Mousa, M. (2014). Systematic review of quality of care in Saudi Arabia. Saudi Medical Journal, 35(8), pp 802-809.

Al-Shahri, M.Z. (2002). Culturally sensitive caring for Saudi patients. Journal of Transcultural Nursing, 13(2), pp 133138.

Alshaikh, Z., Alkhodari, M., Sormunen, T. \& Hilleras, P. (2015). Nurses' knowledge about palliative care in an intensive care unit in Saudi Arabia. Middle East Journal of Nursing, 101(1643), pp 1-7.

Alsulaimani, A.A. (2014). Practical competency of Filipino nurses working in Taif city, Kingdom of Saudi Arabia. Saudi Journal for Health Sciences, 3(2), pp 85-91.

AlYami, M. \& Watson, R. (2014). An overview of nursing in Saudi Arabia. Journal of Health Specialties, 2(1), pp $10-12$.

Bauce, K., Kridli, S. \& Fitzpatrick, J. (2014). Cultural competence and psychological empowerment among acute care nurses. Online Journal of Cultural Competence in Nursing and Healthcare, 4(2), pp 27-38.

Campinha-Bacote, J.C. (2011). Delivering patient-centered care in the midst of a cultural conflict. The Online Journal of Issues in Nursing, 16(2):5

Darnell, L.K. \& Hickson, S. V. (2015). Cultural competent patient-centered nursing care. Nursing Clinics, 50(1), 99108.

De Beer, J. \& Chipps, J. (2014). A survey of cultural competence of critical care nurses in Kwa-Zulu Natal. SAJCC, $30(2), 50-54$.

Felemban, E., O’Connor, M. \& McKenna, L. (2014). Cultural view of nursing in Saudi Arabia. Middle East Journal of Nursing, 8(4), pp 8-14.

Hussein, A. (2014). Relationship between nurses' and physicians' perceptions of organizational health and quality of patient care. Eastern Mediterranean Health Journal, 20(10), pp 634-642.

Harnegie, M.P. (2017). Theories Guiding Nursing Research and Practice: Making Nursing Knowledge Development Explicit edited by Joyce J. Fitzpatrick and Geraldine McCarthy. Journal of Hospital Librarianship, 17:1, pp 89-90.

Ingram R.R. (2012) Using Campinha-Bacote's process of cultural competence model to examine the relationship between health literacy and cultural competence. Journal of Advanced Nursing, 68(3), pp 695-704.

Lamadah, S. \& Sayed, H. Y. (2014). Challenges facing nursing profession in Saudi Arabia. Journal of Biology, Agriculture and Healthcare, 14(7), pp 20-27.

Ministry of Health. (2012). Health statistical yearbook for the year 2011. Riyadh: Ministry of Health in Saudi Arabia.

Noble, A. \& Rom, M. (2014). The effect of a cultural competence educational intervention for first-year nursing 
students in Israel. Journal of Transcultural Nursing, 25(1), 87-94.

Reyes, H., Hadley, L. \& Davenport, D. (2013). A comparative analysis of cultural competence in beginning and graduating nursing students. International Scholarly Research Notices, Article ID 929764, 5.

Rooyen, D.V., Telford, S. \& Strümpher, J. (2010). Nursing in Saudi Arabia: Reflections on the experiences of South African Nurses. Journal of Interdisciplinary Health Sciences, 15(1).

Tucker, C., RoncoronI, J. \& Sanchez, J. (2015). Patient-centered, Culturally Sensitive Health Care. American Journal of Lifestyle Medicine, 9(1), 64-77.

Zakari, N.M., Al Khamis, N.I. \& Hamadi, H.Y. (2010). Conflict and professionalism: perceptions among nurses in Saudi Arabia. International Nursing Review, 57(3), pp 297-304. 